九州大学学術情報リポジトリ

Kyushu University Institutional Repository

\title{
ELISA for the Detection of Marker Compound for Crop Fertilizer Use of Various Medicinal Crop Extracts using Bacterium
}

SON, Tae-Kwon

Department of Farm Management, College of Agriculture and Life Sciences, Kyungpook National University

EGUCHI, Toshihiko

Biotron Application Center, Kyushu University

SHOYAMA, Yukihiro

Department of Pharmacognosy, Faculty of Pharmaceutical Sciences, Nagasaki International University

TANAKA, Hi royuki

Department of Pharmacognosy, Faculty of Pharmaceutical Sciences, Kyushu University

https://doi.org/10.5109/2231630

出版情報：九州大学大学院農学研究院紀要. 64 (1)，pp.27-32，2019-02-28. Faculty of Agriculture， Kyushu University

バージョン :

権利関係 : 


\title{
ELISA for the Detection of Marker Compound for Crop Fertilizer Use of Various Medicinal Crop Extracts using Bacterium
}

\author{
Tae-Kwon SON ${ }^{1}$, Toshihiko EGUCHI, Yukihiro SHOYAMA ${ }^{2}$ and Hiroyuki TANAKA ${ }^{3 *}$ \\ Biotron Application Center, Kyushu University, Fukuoka 819-0395, Japan \\ (Received October 18, 2018 and accepted November 12, 2018)
}

\begin{abstract}
This study builds an easy and quick method of analysing the active ingredients in bio-fertilizers to ensure the uniformity of materials in bio-fertilizers that are made using ingredients that are cruder herbal materials or remnants after the primary medicinal crops are used as herbal materials. An ELISA was created using MAb, and it can substitute for HPLC in the analysis of saikosaponin a (SSa), ginsenoside (G$\mathrm{Rb} 1$ ) and ginsenoside (G-Rg1), which are active ingredients of ginseng, and the ELISA can be used for various ginseng plants grown as typical medicinal crops. The concentration measurement ranges of G-Rb1, G-Rg1, and SSa are $12.5 \sim 250 \mathrm{ng} / \mathrm{mL}, 0.5 \sim 12.5 \mu \mathrm{g} / \mathrm{mL}$, and $0.26 \sim 1.5 \mu \mathrm{g} / \mathrm{mL}$, respectively.
\end{abstract}

Key words: Crop fertilizer, ginseng, Bupleurum species, ginsenoside, saikosaponin, ELISA

\section{INTRODUCTION}

For a long time, Ginseng and Bupeurum falcatum have been the most commonly used medicinal crops in traditional Chinese oriental medicine. The ginsenosides derived from ginseng possess various biological activities (Shibata, 1982; Christensen, 2009). Several reports have shown that ginseng possesses anticancer (Duan et al., 2017), cancer preventing (Yamabe et al., 2013), memory enhancing (Liu et al., 2011), immunomodulatory and antiplatelet aggregating (Shibata, 1982) activities. Additionally, ginseng contains a variety of active ingredients, such as ginsenosides, polysaccharides, flavonoids, volatile oils, amino acids, and vitamins (Wu et al., 2018; Lu et al., 2009).

Generally, these materials are used and then discarded by people, but those remnants or useless materials can be used as biofertilizer through the fermentation process. Examples of bio-fertilizers used for medicinal crops are extremely rare. Bio-fertilizers could in turn stimulate plant growth by increasing the availability and supply of nutrients to the targeted host plants, expanding mushroom yield (Nam et al., 2018) and promoting the stems and leaves of cassava (Luo et al., 2008). Although there have been many reports for the application of crop cultivation using biofertilizers (Nam et al., 2018; Xiong et al., 2017), as in this study, there are few reports on the availability of bio-fertilizer and enzyme-linked immunosorbent assay (ELISA) analysis of fermentation liquid of ginseng and herbal remnants. One of the largest problems that occurs when bio-fertilizer is manufac-

${ }^{1}$ Department of Farm Management, College of Agriculture and Life Sciences, Kyungpook National University, 80, Daehak-ro, Buk-gu, Daegu, 41566, Republic of Korea

2 Department of Pharmacognosy, Faculty of Pharmaceutical Sciences, Nagasaki International University, 2825-7 Huis Ten Bosch Machi, Sasebo City, Nagasaki 859-3298, Japan

${ }^{3}$ Department of Pharmacognosy, Faculty of Pharmaceutical Sciences, Kyushu University, 3-1-1 Maidashi, Higashi-ku, Fukuoka 812-8582, Japan

* Corresponding author (E-mail: htanaka@phar.kyushu-u.ac.jp) tured with medicinal crops is that there is a content gap of secondary metabolites. The content gap of secondary metabolites may occur based on various conditions, including different varieties of ginseng, different cultivation areas and different cultivation methods (Kang et al., 2016; Chung et al., 2017; Kim et al., 2013). Therefore, an easy and fast ELISA is needed to keep the content and intensity of active ingredients constant and to secure a uniformity of raw materials when the bio-fertilizer is manufactured. The successful production of the bio-fertilizer requires solving issues regarding $\mathrm{pH}$ and temperature control to prevent unstable quality management (Liu, 2005). A uniformity of the active ingredients or quality management is one of the most important factors in the final stage. Many researchers currently analyse the active ingredients through an HPLC method, but ELISA is one way to analyse many samples simultaneously within a short time. ELISA analysis has been reported for total ginsenosides in ginseng (Morinaga et al., 2006); quantitative analysis of ginsenosides by ELISA using monoclonal antibodies has been reported in American ginseng berry and flower samples (Sritularak et al., 2009); the identification of Panax species has been reported using ELISA, RAPD and eastern blotting (Tanaka et al., 2006); and indirect competitive ELISA has been reported for determining a distinctive saponin from Panax notoginseng (Limsuwanchote et al., 2014).

This research will measure saikosaponin a (SSa), ginsenoside $\mathrm{G}-\mathrm{Rb} 1$ (G-Rb1) and ginsenoside Rg1 (GRg1) contents, which are typical ingredients among various secondary metabolites that affect the quality of the biofertilizer during manufacture. The ELISA assays reported here will be easy and quick methods of component measurement to promote the uniformity of product quality when bio-fertilizer is manufactured, and they will contribute to the production of stable products when bio-fertilizer is manufactured by improving the uniformity of the raw materials used to produce the bio-fertilizer. 


\section{MATERIALS AND METHODS}

\section{Chemicals and Immunochemicals}

Ginsenosides and saikosaponins were purchased from Wako Pure Chemical Ind., Ltd. Bovine serum albumin (BSA) and human serum albumin (HSA) were obtained from Pierce (Rockford, IL, USA). Peroxidase-labeled anti-mouse IgG was obtained from ICN Pharmaceuticals., Inc. (USA). All other chemicals were standard commercial products of analytical grade. The roots of ginsengs and Bupleurum species were purchased from Nakai Koshindo Co., Ltd. (Kobe, Japan)

\section{Sample Preparation}

Dried samples (20 mg) of various ginsengs and Bupleuri radix were powdered, extracted with $\mathrm{MeOH}$ $(0.5 \mathrm{ml})$ under sonication 5 times, filtered, and then evaporated. For the elimination of a malonyl group of malonyl ginsenosides in ginseng and an acyl group from acylated saikosaponins in Bupleuri radix, the extracts were treated with $5 \%$ of $\mathrm{KOH}$ in $\mathrm{MeOH}$ at room temperature for $2 \mathrm{hr}$, then neutralized with $1 \mathrm{M} \mathrm{HCl}$ in $\mathrm{MeOH}$, and assayed by ELISA.

\section{Competitive ELISA}

G-Rb1-HSA (5 molecules of G-Rb1 per molecule of HSA) $(100 \mu \mathrm{L}, 1 \mu \mathrm{g} / \mathrm{mL})$ was adsorbed to the wells of a 96 well-immunoplate and then treated with $300 \mu \mathrm{L}$ SPBS for $1 \mathrm{hr}$ to reduce non-specific adsorption. Fifty $\mu \mathrm{l}$ of various concentrations of G-Rb1 dissolved in 20\% of $\mathrm{MeOH}$ solution was incubated with $50 \mu \mathrm{l}$ (IgG: $1.0 \mu \mathrm{g} /$ $\mathrm{mL}$ ) of anti-G-Rb1 MAb solution for $1 \mathrm{hr}$. The plate was washed three times with TPBS, and then the MAb was combined with $100 \mu \mathrm{L}$ of a 1:1000 dilution of peroxidase-labeled anti-mouse IgG for $1 \mathrm{hr}$. After washing the plate three times with TPBS, $100 \mu \mathrm{L}$ of ABTS solution was added to each well and incubated for $15 \mathrm{~min}$. Absorbance was measured by the micro plate reader at $405 \mathrm{~nm}$. Competitive ELISAs for G-Rg1 and SSa were set up in the same manner as that for G-Rb1.

\section{HPLC Instruments and Conditions for G-Rb1 and G-Rg1}

The chromatograph used in this study consisted of a LC-10AD equipped with an SPD-10A vp UV detector (Shimadzu, Kyoto, Japan) and a Chromatopac C-R8A data analyzer system (Shimadzu, Kyoto, Japan). The analytical column was a Cosmosil 5 C18-AR column (4.6×150 mm i.d., Nacalai Tesque, Kyoto, Japan) maintained at room temperature. The mobile phase for G-Rb1 was $\mathrm{CH}_{3} \mathrm{CN}$-water (3:7, by volume) containing $50 \mathrm{mM}$ $\mathrm{KH}_{2} \mathrm{PO}_{4}$. The mobile phase for $\mathrm{G}-\mathrm{Rg} 1$ was $\mathrm{CH}_{3} \mathrm{CN}$-water (1:4, by volume) containing $50 \mathrm{mM} \mathrm{KH}_{2} \mathrm{PO}_{4}$. The flow rate was $1.0 \mathrm{~mL} / \mathrm{min}$. UV absorbance detection at $202 \mathrm{~nm}$ was used for monitoring the effluent.

\section{HPLC Instruments and Conditions for SSa}

HPLC analysis was performed on a Model LC-10AD (Shimadzu Co., Ltd.) Pump connected with TSK-gel ODS-120A (4.6 I.D. $\times 250 \mathrm{~mm}$. Tosoh Co., Ltd.), equipped with a UV-8 Model II Spectrophotometer (Tosoh Co., Ltd.) detected at $203 \mathrm{~nm}$. The mobile phase was $50 \mathrm{mM} \mathrm{KH}_{2} \mathrm{PO}_{4}$ buffer containing $44 \%$ of $\mathrm{CH} 3 \mathrm{CN}$.

\section{Correlation between HPLC and ELISA Analysis}

The interpolated concentrations of the samples obtained by each method were then compared by linear regression analysis.

\section{Bio-fertilizer Sample}

The bio-fertilizer sample was prepared for active ingredient analysis of SSa, G-Rb1 and G-Rg1 contents in liquid materials before and after fermentation using the waste products or residues of ginseng and Bupleurum falcatum after their medicinal use. The fermentation method followed the patent (PCT/KR2016/008158).

\section{RESULTS}

\section{Development of the analysis method for G-Rb1 and G-Rg1 by ELISA}

Tables 1 and 2 show the cross-reactivities of antiGb1 MAb 9G7 and anti-Gg1 MAb 1F4, respectively. Tables 1 and 2 show the development of an ELISA by indirect competition using anti-Gb1 MAb 9G7 and antiGg1 MAb 1F4, respectively, which showed cross-reactivities. First, we developed a calibration curve using protein standards of G-Rb1 and G-Rg1. As a result, the absorbance decreased depending on the concentration as in Figs. 1 and 2, and there was a straight-line relationship between the concentration and absorbance of ginsenoside. G-Rb1 was linear between 12.5 and $250 \mathrm{ng} /$ $\mathrm{mL}$ of the concentration curve, and G-Rg1 was linear between 0.5 and $12.5 \mu \mathrm{g} / \mathrm{mL}$ of the concentration curve. We recognized that establishing an ELISA was possible by measuring the concentration ranges of G-Rb1 and GRg1. Table 3 shows differences between G-Rb1 in ELISA and HPLC measurement values using various ginseng samples - Ginseng (P. ginseng), Red Ginseng, Fibrous

Table 1. Cross-reactivities of anti-G-Rb1 MAb 9G7

\begin{tabular}{ll}
\hline Compound & Cross-reactivity (\%) \\
\hline Ginsenoside Rb1 & 100 \\
Ginsenoside Rc & 0.024 \\
Ginsenoside Rd & 0.020 \\
Ginsenoside Re & $<0.005$ \\
Ginsenoside Rg1 & $<0.005$ \\
Glycyrrhizin & $<0.005$ \\
Digitonin & $<0.005$ \\
Solamargine & $<0.005$ \\
Solasonine & $<0.005$ \\
$\beta$-Sitosterol & $<0.005$ \\
Cholesterol & $<0.005$ \\
Deoxycholic acid & $<0.005$ \\
Ergosterol & $<0.005$
\end{tabular}


Table 2. Cross-reactivities of anti-G-Rg1 MAb 1F4

\begin{tabular}{ll}
\hline Compound & Cross-reactivity (\%) \\
\hline Ginsenoside Rg1 & 3.3 \\
Ginsenoside Re & $<0.93$ \\
Ginsenoside Rb1 & $<0.93$ \\
Ginsenoside Rc & $<0.93$ \\
Ginsenoside Rd & $<0.93$ \\
Glycyrrhizin & $<0.93$ \\
Digitonin & $<0.93$ \\
Solamargine & $<0.93$ \\
Solasonine & $<0.93$ \\
$\beta$-Sitosterol & $<0.93$ \\
Cholesterol & $<0.93$ \\
Deoxycholic acid & $<0.93$ \\
Ergosterol &
\end{tabular}

Ginseng, San-chi Ginseng (P. notoginseng), American Ginseng (P. quinquefolium), and Japanese Ginseng ( $P$. japonicus). The results of Ginseng ( $P$. ginseng) showed that the ELISA measurement was $3.63 \pm 0.37 \mu \mathrm{g} / \mathrm{mg}$ dry wt. and that the HPLC measurement was $4.83 \pm 0.40 \mu \mathrm{g} /$ mg dry wt.. Red Ginseng was $5.90 \pm 0.17 \mu \mathrm{g} / \mathrm{mg}$ dry wt. from ELISA and 5.36 \pm 0.41 from HPLC. Fibrous Ginseng was $64.44 \pm 3.64 \mu \mathrm{g} / \mathrm{mg}$ dry wt. from ELISA and $69.75 \pm$ $1.45 \mu \mathrm{g} / \mathrm{mg}$ dry wt. from HPLC. As a result, most ginseng samples showed that the values obtained by ELISA and HPLC were similar. Table 4 measured the Ginsenoside Rg1 content from ELISA and HPLC by using various ginseng samples - Ginseng (P. ginseng), Red Ginseng, Fibrous Ginseng, San-chi Ginseng ( $P$. notoginseng), American Ginseng ( $P$. quinquefolium), Japanese Ginseng (P. japonicus), and compared correlation values between ELISA and HPLC. The result of Ginseng (P. ginseng) showed that the measurement obtained by ELISA was $2.28 \pm 0.02 \mu \mathrm{g} / \mathrm{mg}$ dry wt. and that the measurement obtained by HPLC was $1.78 \pm 0.07 \mu \mathrm{g} / \mathrm{mg}$ dry wt.. Red Ginseng was $1.34 \pm 0.08 \mu \mathrm{g} / \mathrm{mg}$ dry wt. from ELISA and $1.65 \pm 0.05 \mu \mathrm{g} / \mathrm{mg}$ dry wt. from HPLC. Fibrous Ginseng was $4.98 \pm 0.04 \mu \mathrm{g} / \mathrm{mg}$ dry wt. from ELISA and $5.39 \pm 0.25 \mu \mathrm{g} / \mathrm{mg}$ dry wt. from HPLC. As a result, most ginseng samples showed that the values obtained by ELISA and HPLC were similar, such as they had been for

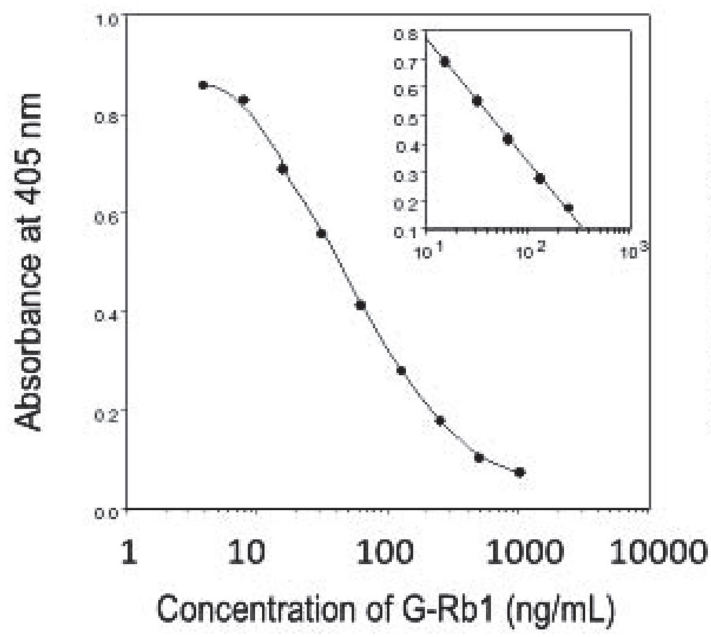

Fig. 1. Calibration curve for G-Rb1 in ELISA.

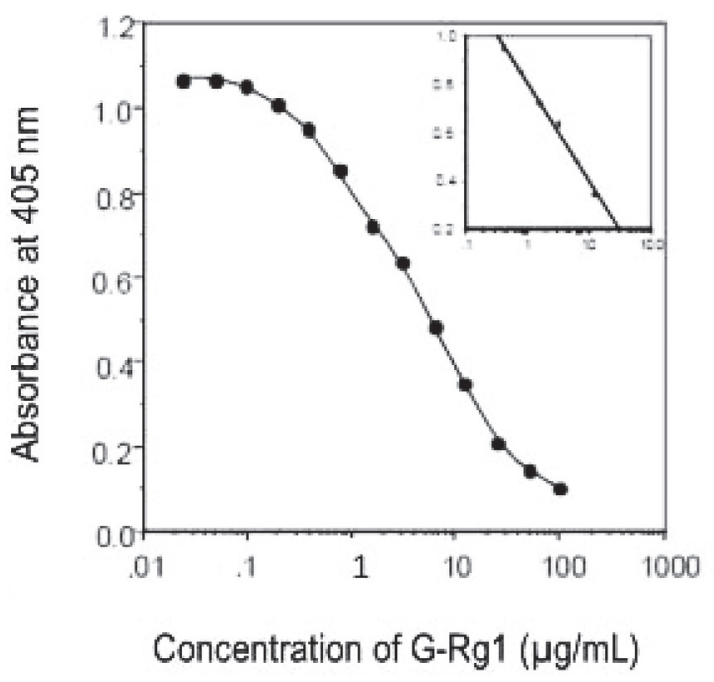

Fig. 2. Calibration curve for G-Rg1 in ELISA.

ginsenoside Rb1. Measuring the G-Rb1 and G-Rg1 contents in various ginseng samples by ELISA and HPLC was a success (Tables 3 and 4). The values obtained by ELISA and HPLC are similar, verifying that the ELISA method is reliable.

Table 3. Ginsenoside Rb1 content of various ginseng determined by ELISA

\begin{tabular}{lcc}
\hline \multirow{2}{*}{ Sample (after alkaline treatment) } & Content ( $\mu$ g/mg dry wt.) \\
\cline { 2 - 3 } & ELSA & HPLC \\
\hline Ginseng (P. ginseng) & $3.63 \pm 0.37$ & $4.83 \pm 0.40$ \\
Red ginseng (P. ginseng) & $5.90 \pm 0.17$ & $5.36 \pm 0.41$ \\
Fibrous ginseng (P. ginseng) & $64.44 \pm 3.64$ & $69.75 \pm 1.45$ \\
San-chi ginseng (P. notoginseng) & $47.51 \pm 3.34$ & $42.39 \pm 1.39$ \\
American ginseng (P. quinquefolium) & $48.51 \pm 1.79$ & $47.96 \pm 1.04$ \\
Japanese ginseng (P. japonicus) & $1.37 \pm 0.34$ & $0.63 \pm 0.06$ \\
\hline
\end{tabular}


Table 4. Ginsenoside Rg1 content of various ginseng determined by ELISA

\begin{tabular}{lcr}
\hline Sample (after alkaline treatment) & Content ( $\mu$ g/mg dry wt.) \\
\cline { 2 - 3 } Ginseng (P. ginseng) & $2.28 \pm 0.02$ & HPLC \\
Red ginseng (P. ginseng) & $1.34 \pm 0.08$ & $1.65 \pm 0.07$ \\
Fibrous ginseng (P. ginseng) & $4.98 \pm 0.04$ & $5.39 \pm 0.25$ \\
San-chi ginseng (P. notoginseng) & $22.9 \pm 3.20$ & $25.93 \pm 0.32$ \\
American ginseng (P. quinquefolium) & $3.15 \pm 0.23$ & $2.49 \pm 0.04$ \\
Japanese ginseng (P. japonicus) & $0.12 \pm 0.01$ & - \\
\hline
\end{tabular}

\section{Development of the analysis method for SSA by ELISA}

Table 5 tried to develop ELISA by indirect competition using MAb that showed cross-reactivities. SSa showed a high cross-reactivity with all antibodies that were used, such as 1G6, 3G10, 2A8, and 4B9. However, SSb2, SSc, and SSd showed a little cross-reactivity depending on the antibodies, but these components did not have high reliability in their cross-reactivities. We wrote a calibration curve by using a standard SSa sample as shown in Fig. 3. As a result, the absorbance decreased depending on the concentration, as in Fig. 3, and there was a straight-line relationship between the concentration and absorbance of SSa. SSa was linear between 0.26 and $1.5 \mu \mathrm{g} / \mathrm{mL}$ of the concentration curve, and we recognized that it was possible to establish an ELISA that measures the concentration ranges. Fig. 4 represents the measurement results from ELISA and

Table 5. Cross-reactivities of anti-SSa MAb 1G6

\begin{tabular}{lrccc}
\hline \multicolumn{1}{c}{ Compound } & $1 \mathrm{G} 6$ & $3 \mathrm{G} 10$ & $2 \mathrm{~A} 8$ & $4 \mathrm{~B} 9$ \\
\hline Saikosaponin a & 100 & 100 & 100 & 100 \\
Saikosaponin b2 & $<0.25$ & 63.77 & 2.92 & 10.58 \\
Saikosaponin c & 2.65 & 28.52 & 6.31 & 49.23 \\
Saikosaponin d & 3.76 & 15.62 & 3.69 & 20.89 \\
Glycyrrhizin & $<0.25$ & $<0.25$ & $<0.25$ & $<0.25$ \\
Digitonin & $<0.25$ & 1.42 & $<0.25$ & $<0.25$ \\
Solasonine & $<0.25$ & $<0.25$ & $<0.25$ & $<0.25$ \\
$\beta$-Sitosterol & $<0.25$ & $<0.25$ & $<0.25$ & $<0.25$ \\
Cholesterol & $<0.25$ & $<0.25$ & $<0.25$ & $<0.25$ \\
Deoxycholic acid & 0.45 & $<0.25$ & $<0.25$ & $<0.25$ \\
Ergosterol & $<0.25$ & $<0.25$ & $<0.25$ & $<0.25$ \\
\hline
\end{tabular}

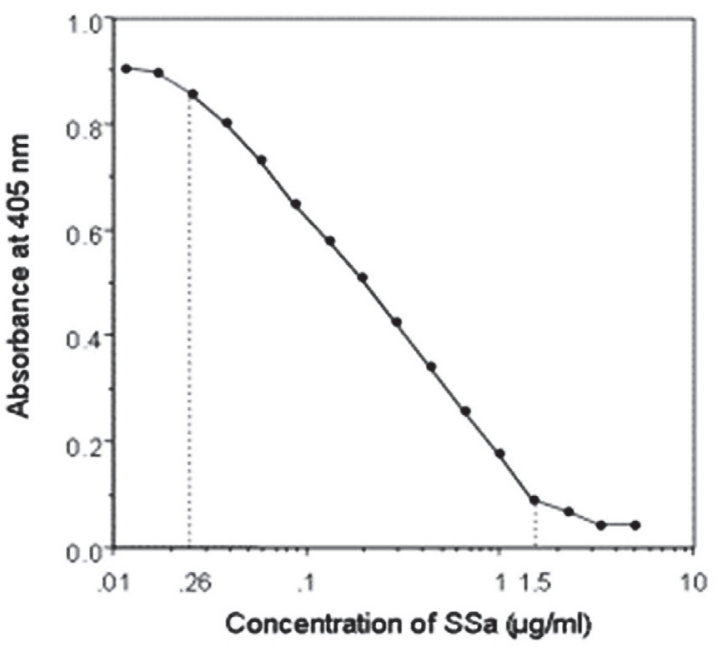

Fig. 3. Calibration curve for SSa in ELISA.

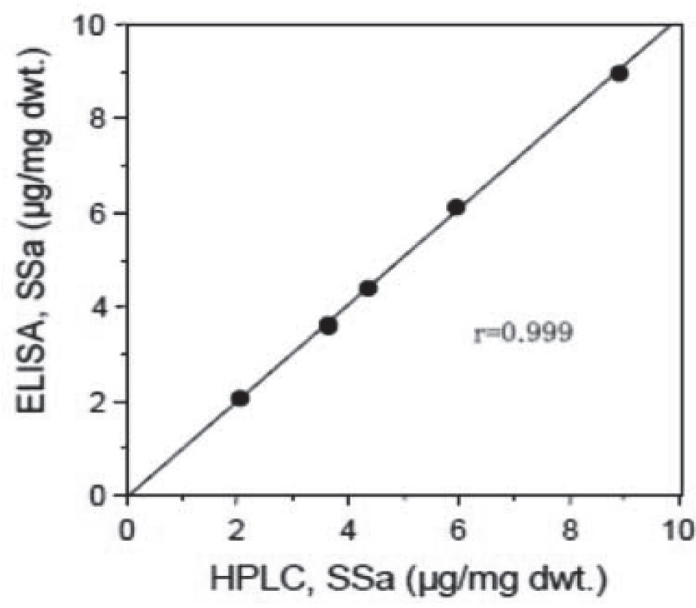

Fig. 4. Comparison of SSa contents determined by ELISA and HPLC system.

Table 6. SSa, G-Rb1 and G-Rg1 contents in Bio-fertilizer using liquid residues of ginseng and Bupleurum falcatum before and after fermentation

\begin{tabular}{cccccccc}
\hline & \multicolumn{5}{c}{ Content $(\mu \mathrm{g} / \mathrm{mL}$ liquid wt.) } \\
\cline { 2 - 7 } Samples & \multicolumn{5}{c}{ ELISA } & \multicolumn{3}{c}{ HPLC } \\
\cline { 2 - 7 } & SSa & G-Rb1 & G-Rg1 & SSa & G-Rb1 & G-Rg1 \\
\hline Before fermentation & 1.12 & 0.62 & 0.57 & 1.18 & 0.61 & 0.58 & 0.67 \\
After fermentation & 1.33 & 0.78 & 0.59 & 1.39 & 0.80 & 0.67 \\
\hline
\end{tabular}


HPLC using a Bupleurum falcatum sample, which is one of the medicinal ginseng crops. As a result, we could measure SSa content by using a Bupleurum falcatum sample, as in Fig. 4, and the values of ELISA and HPLC show a high correlation $(\mathrm{r}=0.999)$.

Table 6 shows SSa, G-Rb1 and G-Rg1 contents in bio-fertilizer using liquid residues of ginseng and Bupleurum falcatum before and after fermentation.

\section{DISCUSSION}

Many applications researching ELISAs for traditional Chinese herbal medicines or crude drugs have been reported after the first report the immunoassay system of G-Rb1 of Tanaka et al. (1999). Fukuda et al. (2000) applied qualitative and quantitative surveys of ginsenoside $\mathrm{Rb} 1$ and related ginsenosides in roots and traditional Chinese herbal medicines using a combination of ELISAs, Western blotting and immunoaffinity concentration. The contents of G-Rg1, G-Rb1 and SSa in various crude drugs were analysed by the competitive ELISA and HPLC methods described above (Table 3 and 4, Fig. 4). Kitagawa et al. (1987), Samukawa et al. (1995) and Xuan et al. (1999) analysed the ginsenoside contents in various crude ginsengs by HPLC. Our results were in good agreement with their previous reports as described above. The newly established ELISA was more sensitive than the HPLC methods (Sticher and Soldati 1979; Soldati and Sticher 1980). The correlation coefficient was calculated from fitting a straight line analysed by ELISA and HPLC methods. There was a good correlation $(\mathrm{r}=0.999)$ between the assay values obtained by the two methods (Fig. 1, 2, 3). This methodology can be utilized for the assay of G-Rg1, G-Rb1 and SSa; therefore, it is possible to study a large number of samples in vitro as well as various samples in the field (Shoyama et al., 1995, 1997).

Fermentation is the key in the process of producing bio-fertilizer. However, in current production, most factories use simple manually operated instruments to measure $\mathrm{pH}$ values and temperature; thus, $\mathrm{pH}$ and temperature fluctuations and quality consistency become severe problems (Liu et al., 2005). The final step will minimize the problem of inconsistent quality by adjusting the active ingredients. To resolve the quality consistency problem, the method of detection of the active ingredients should be simple and rapid. Therefore, this study applied ELISA to G-Rg1, G-Rb1 and SSa measurements by using various ginseng samples to ensure uniformity of the quality of the bio-fertilizer. The contents of G-Rg1, $\mathrm{G}-\mathrm{Rb} 1$, and SSa from various ginseng and Bupleurum falcatum samples as determined by ELISA and HPLC were similar, and a calibration curve showed a high correlation between these methods. Therefore, these results support the reliability of the ELISA method.

To stabilize the quality of bio-fertilizer produced using medicinal crop materials, it is necessary to test the active ingredients, and as shown in Table 6, we succeeded in constructing a standard substance detection method using an ELISA. Zhang et al. (2008) indicated that the selected bacterial strain and the produced biofertilizer showed good effectiveness in improving the soil fertility and growth of tea plants and increased the yield of tea gardens. Bio-fertilizer has good prospects in both organic food production and safe food production. Biofertilizer has been used for various growing crops, including citrus (Liu et al., 2011) and tea plants (Zhang et al., 2008), not only for medicinal crops. There is a potential application for ELISAs in the manufacture of various bio-fertilizers. In conclusion, ELISAs could be used to survey low concentrations of G-Rb1, G-Rg1 and $\mathrm{SSa}$ in samples of plant origin and/or in experimental fields anywhere. Moreover, this assay can be used to detect low concentrations of active ingredients in biofertilizer production.

Maintaining a uniform concentration of extracts originated from medicinal plant by-products is essential for obtaining certain effects when used as a crop fertilizer. At the stage where such extracts are used, concentration measurement is very important. This paper has established a quick and simple method for detecting marker compounds in either crop fertilizers using various medicinal crop extracts or in bio-fertilizers using ELISA.

\section{ACKNOWLEDGEMENTS}

This work was supported by the Korea Institute of Planning and Evaluation for Technology in Food, Agriculture, Forestry (IPET) through the Technology Commercialization Support Program and Advanced Production Technology Development Program, funded by the Ministry of Agriculture, Food and Rural Affairs (MAFRA) (grant numbers: 817022-3 and 112044-3).

\section{REFERENCES}

Christensen L. P. 2009 Ginsenosides chemistry, biosynthesis, analysis, and potential health effects. Advances in Food and Nutrition Research, 55: 1-99

Chung I. M., T. J. Lee, Y. T. Oh, G. B. Kumar, I. B. Jang and S. H. Kim 2017 Ginseng authenticity testing by measuring carbon, nitrogen, and sulfur stable isotope compositions that differ based on cultivation land and organic fertilizer type. Journal of Ginseng Research, 41(2): 195-200

Duan Z., J. Deng, Y. Dong, C. Zhu, W, Li, D. Fan 2017 Anticancer effects of ginsenoside Rk3 on non-small cell lung cancer cells in vitro and in vivo. Food \& Function, 8(10): 3723-3736

Fukuda N., H. Tanaka and Y. Shoyama 2000 Applications of ELISA, western blotting and immunoaffinity concentration for survey of ginsenosides in crude drugs of Panax species and traditional chinese herbal medicines. Analyst, 125: 1425-1429

Kang, H. M., S. J. Cho, S. I. Choi, N. Sato and H. Kim 2016 Revitalizing mountain mginseng cultivation in north jeolla province. Small-scale Forestry, 15(4): 497-516

Kim S. H., M. Y. Kim, S. B. Lee, I. B. Jung and K. S. Lee 2013 Improvement of shade structures for ginseng cultivation. Journal of Biosystems Engineering, 38(4): 270-278

Kitagawa I., T. Taniyama, H. Shibuya, T. Noda and M. Yoshikawa 1987 Chemical studies on crude drug processing. V. On the constituents of ginseng radix rubra (2): Comparison of the constituents of white ginseng and red ginseng prepared from the same Panax ginseng root. Yakugaku Zasshi, 107: 495-505 
Limsuwanchote S., J. Wungsintaweekul, G. Yusakul, J. Y. Han, S. T. Kaori, H. Tanaka, Y. Shoyama and S. Morimoto 2014 Preparation of a monoclonal antibody against notoginsenoside R1, a distinctive saponinfrom Panax notoginseng, and its application to indirect competitive ELISA. Planta Medica, 80(4): 337-342

Liu L., J. Huang, X. Hu, K. Li and C. Sun 2011 Simultaneous determination of ginsenoside(G-Re, G-Rg1, G-Rg2, G-F1, GRh1) and protopanaxatriol in human plasma and urine by LCMS/MS and its application in a pharmacokinetics study of G-Re in volunteers. J. of Chromatography. 879(22): 2011-2017

Liu Z. 2011 Production of bio-organic fertilizer by bio-fermentation of citrus residue. Journal of Anhui Agricultural Sciences, 39(20): 12176-12178

Liu Z., D. Feng, W. Zhou, C. Bi, D. Han and Y. Xu 2005 Application of $\mathrm{pH}$-temperature intelligent control system for fermentation of bio-fertilizer. Transactions of the Chinese Society of Agricultural Engineering. 21(8): 187-189

Lu J. M., Q. Yao and C. Chen 2009 Ginseng compounds: an update on their molecular mechanisms and medical applications. Curr Vasc Pharmacology, 7(3): 293-302

Luo, X., Z. Cen, H. Xie, Y. Pan, C. Liao, Z. Shao, H. Chen 2008 Effects of bio-organic fertilizer on the growth of cassava and the physical and chemical biological character of soil. Acta agriculturae boreali-occidentalis sinica, 17(1): 167-173

Morinaga O., H. Tanaka and Y. Shoyama 2006 Enzyme-linked immunosorbent assay for the determination of total ginsenosides in ginseng. Analytical Letters, 39(2): 257-296

Nam W. L., X. Y. Phang, M. H. Su, R. K. Liew, N. L. Ma, M. H. N. B. Rosli and S. S. Lam 2018 Production of bio-fertilizer from microwave vacuum pyrolysis of palm kernel shell for cultivation of Oyster mushroom (Pleurotus ostreatus). The Science of the Total Environment, 624: 9-16

Samukawa K, H. Yamashita, H. Matsuda and M. Kubo 1995 Simultaneous analysis of ginsenosides of various ginseng radix by HPLC. Yakugaku Zasshi, 115: 241-249

Shibata S. 1982 Chemistry of components in ginseng. $J$. Traditional Sino-Japanese Medicine, 3: 62-69

Shoyama Y., H. Matsushita, X. X. Zhu and H. Kishira 1995 Somatic embryogenesis in ginseng (Panax species). Bajaj YPS (ed.). Biotechnology in Agriculture and Forestry, $\mathbf{3 1}$ 343-356
Shoyama Y, X. X. Zhu, R. Nakai and S. Shiraishi 1997 Micropropagation of Panax notoginseng by somatic embryogenesis and RAPD analysis of regenerated plantlets. Plant Cell Rep, 16: 450-453

Soldati F. and O. Sticher 1980 HPLC separation and quantitative determination of ginsenosides from Panax ginseng, Panax quinquefolium and from ginseng drug preparations. 2nd communication. Planta Medica, 39: 348-357

Sritularak B., O. Morinaga, Y. Chun-Su, S. H. Yuan, Y. Shoyama and H. Tanaka 2009 Quantitative analysis of ginsenosides Rb1, Rg1, and Re in Americanginseng berry and flower samples by ELISA using monoclonal antibodies. Journal of Natural Medicines, 63(3): 360-363

Sticher O. and F. Soldati 1979 HPLC separation and quantitative determination of ginsenosides from Panax giseng, Panax quinquefolium and from ginseng drug prepararions. Planta Medica, 36: 30-42

Tanaka H., N. Fukuda and Y. Shoyama 1999 Formation of monoclonal antibody against a major ginseng component, ginsenoside Rb1 and its characterization. Cytotechnology, 29: 115-120

Tanaka H., N. Fukuda and Y. Shoyama 2006 Identification and differentiation of Panax species using ELISA, RAPD and eastern blotting. Phytochemical Analysis, 17(1): 46-55

Wu W., C. Jiao, H. Li, Y. Ma, L. Jiao and S. Liu 2018 LC-MS based metabolic and metabolomic studies of Panax ginseng. Phytochemical Analysis, 29: 331-340

Xiong W. G, S. J. Alexandre, Q. Zhao, H. Wu, R. Li, G. A. Kowalchuk and Q. Shen 2017 Bio-fertilizer application induces soil suppressiveness against Fusarium wilt disease by reshaping the soil microbiome. Soil Biology \& Biochemistry, 114: 238-247

Xuan L. J., H. Tanaka, Y. Xu and Y. Shoyama 1999 Preparation of monoclonal antibody against crocin and its characterization. Cytotechnology, 29: 65-70

Yamabe N., Y. J. Kim, S. Lee, E. J. Cho, S. H. Park, J. Ham, H. Y. Kim and K. S. Kang 2013 Increase in antioxidant and anticancer effects of ginsenoside re-lysine mixture by maillard reaction. Food Chemistry, 138(2-3): 876-883

Zhang Y., C. Shuoqi, L. Hongyan, F. Haiping and L. Jianquan 2008 Study on nutritional function of bacterial bio-fertilizer on tea plant. Journal of Tea Science, 28(2): 123-128 